\title{
In vitro Antimicrobial Synergy Testing of Extensively Drug-Resistant Clinical Isolates at an Organ Transplant Center in Nepal
}

\author{
Rashmi Karki (iD) 1,2,* \\ Samir Lamichhane ${ }^{3, *}$ \\ Buddha Bahadur Basnet ${ }^{4}$ \\ Anuja Dahal ${ }^{5}$ \\ Bal Krishna Awal 2,6 \\ Shyam Kumar Mishra (D) ${ }^{7,8, *}$ \\ 'Janamaitri Foundation Institute of Health \\ Sciences, Tribhuvan University, Nepal; \\ ${ }^{2}$ National Public Health Laboratory, \\ Kathmandu, Nepal; ${ }^{3}$ Department of \\ Pharmacology, Maharajgunj Medical \\ Campus, Institute of Medicine, Tribhuvan \\ University, Nepal; ${ }^{4}$ Faculty of Science, \\ Nepal Academy of Science and \\ Technology, Lalitpur, Nepal; ${ }^{5}$ Department \\ of Pharmacy, Maharajgunj Medical \\ Campus, Institute of Medicine, Tribhuvan \\ University, Nepal; ${ }^{6}$ Shahid Dharmabhakta \\ National Transplant Center, Bhaktapur, \\ Nepal; ${ }^{7}$ School of Optometry and Vision \\ Science, Faculty of Medicine and Health, \\ UNSW, Sydney, NSW, Australia; \\ ${ }^{8}$ Department of Microbiology, \\ Maharajgunj Medical Campus, Institute of \\ Medicine, Tribhuvan University, Nepal
}

*These authors contributed equally to this work

Correspondence: Shyam Kumar Mishra School of Optometry and Vision Science, UNSW, Sydney, NSW, Australia

$\mathrm{Tel}+61415538329$

Email s.mishrabaishnab@unsw.edu.au
Purpose: Inappropriate use of broad-spectrum antibiotics contributes to the emergence of multidrug-resistant (MDR) bacteria. Finding novel antimicrobial agents and strategies based on synergistic combinations are essential to combat MDR infections. This study was designed to determine in vitro synergy of different antimicrobials against extensively drugresistant (XDR) Gram-negative clinical isolates.

Methods: A descriptive, cross-sectional study was conducted at Human Organ Transplant Center, Nepal, for five months. Clinical isolates were checked for their drug-resistance properties including extended-spectrum beta-lactamase- (ESBL-) and metallo-betalactamase- (MBL-) production. The XDR isolates were further tested for antimicrobial synergy, and the results were interpreted as synergistic, additive, indifferent or antagonistic determining fractional inhibitory concentration of the antibiotics.

Results: Out of total 1155 clinical samples, 308 showed significant growth. Escherichia coli was the most common isolate $(\mathrm{n}=142)$ followed by Klebsiella pneumoniae, Acinetobacter calcoaceticus baumannii (Acb) complex, Pseudomonas aeruginosa and miscellaneous bacteria. Out of the culture positive isolates, $21.4 \%$ were MDR and $10.06 \%$ were XDR. The XDR population comprised K. pneumoniae (18.42\%), E. coli $(9.86 \%)$, Acb complex $(7.41 \%)$ and P. aeruginosa (4.17\%). Among the culture positive isolates, $4.5 \%$ and $5.8 \%$ were ESBLand MBL-producers, respectively. Colistin, polymyxin B, and tigecycline were the antibiotics effective in majority of MDR isolates as compared to carbapenems. The combination of antibiotics - meropenem and colistin showed the highest proportion of "synergy" among all XDR E. coli whereas the combination of amikacin and colistin showed synergistic effect in XDR K. pneumoniae.

Conclusion: A significant proportion of isolates were MDR among which a large fraction was XDR. The combination of meropenem, amikacin and colistin with one another in pair showed beneficial activity in vitro. Such combinations can be utilized as effective therapy for XDR infections. Further studies are required to confirm these findings, and accordingly treatment protocols should be developed in the management of such infections.

Keywords: antimicrobial synergy, ESBL, MBL, MDR, XDR

\section{Introduction}

Antimicrobials are among the most important groups of drugs since the history of medicine. ${ }^{1}$ However, the abundant and inappropriate use of antibiotics have led to the emergence of antimicrobial resistance (AMR) that has progressively been threatening our ability to treat infections. ${ }^{2,3}$ Resistance even to the most powerful classes of antibiotic is emerging among various bacteria, rendering the therapies 
more precarious, costlier, or even unsuccessful and hence AMR has become one of the principal public health problems of the $21^{\text {st }}$ century. ${ }^{2-4}$

A variety of bacterial enzymes responsible for mediating drug resistance in Gram-negative bacteria has been identified including extended-spectrum $\beta$-lactamases (ESBLs), AmpC $\beta$-lactamases, carbapenemases like Klebsiella pneumoniae carbapenemase (KPC), Metallo- $\beta$ lactamases (MBLs), and OXA-48-like-carbapenemase in various parts of the world. ${ }^{5-9}$ Various strategies have been adopted since the advent of AMR among these bacteria. Apart from promoting precautionary measures and strictly adhering to the established infection control practices, finding novel antimicrobial agents might be the ultimate strategy to combat these resistant bugs but it takes a huge amount of resources and time to develop one. Hence, optimizing our use of current antimicrobials and strategies based on synergistic combinations have become essential to battle these resistant bacteria. ${ }^{10,11}$

When two or more antibiotics are combined and used simultaneously to treat an infection, three responses are possible. First, the "Antagonistic effect", where the potency of the combination is less than the combined potencies of each antibiotic or even less than the more active single agent; second, the "Additive effect", where the potency of an antibiotic combination is roughly equal to the combined potencies of each antibiotic singly; and third, the "Synergistic effect", where the applied antibiotics work together to produce an effect more potent than the combined potencies of each antibiotic applied singly. ${ }^{12,13}$ Combination therapies are commonly employed, considering their possibility of synergistic effect despite little laboratory guidance as to the efficacy of this approach. ${ }^{14}$ Combination therapy could be a better option than high-dose monotherapy as well, for the reason that it also decreases the dose toxicity to the patient. ${ }^{15}$

In recent years, several antibiotic synergy modules have also been tested in vitro and are being used in vivo as well to manage infections caused by various multidrugresistant (MDR) and extensively drug-resistant (XDR) bacteria. ${ }^{16-18}$ The World Health Organization (WHO) has presented the list of MDR bacteria under three categories according to the urgency of the need for new antibiotics: critical, high, and medium priority. ${ }^{19}$ To our knowledge, there are no published studies on antibiotic synergy conducted in Nepal. Therefore, this study was conducted to determine in vitro synergy of different antimicrobials against XDR Gram-negative clinical isolates that fall under the critical group at a tertiary care hospital in Nepal.

Different laboratory methods are used to assess the activity of antimicrobial combinations. It includes checkerboard titration method, broth method, agar dilution method, diffusion method, saline dilution method, timekill assay, epsilometer (e-strip) test also known as gradient diffusion, etc. In this study, we used the double-disk synergy test and the E-test MIC:MIC method for different antibiotic combinations to determine the different combined effects of antibiotics. As colistin (CL) is one of the last-line agents that has been used for MDR and XDR cases, and various microorganisms are now found to be resistant even to $\mathrm{CL}$, other commonly used antimicrobials, viz., ciprofloxacin (CIP), amikacin (AK) and meropenem (MEM) were used along with $\mathrm{CL}$ to see the potential synergy between such combinations. These combinations are based on standard guideline ${ }^{20}$ which is modified based on the availability of the strips for this study.

\section{Materials and Methods}

A descriptive, cross-sectional study was conducted from August to December 2018 at the Department of Laboratory, Human Organ Transplant Center (HOTC), Bhaktapur. Different clinical specimens (urine, sputum, and blood) from the inpatients were collected aseptically and processed for culture and sensitivity following standard procedure. $^{21}$ The isolates showing significant growth were tested for antimicrobial susceptibility by Kirby-Bauer disk diffusion method on Mueller-Hinton agar (MHA) (HiMedia, India) and interpreted following Clinical and Laboratory Standards Institute (CLSI) 2016 guideline. $^{22}$ The following antibiotic discs (HiMedia, India) were used: amoxicillin $(30 \mu \mathrm{g})$, chloramphenicol $(30 \mu \mathrm{g})$, ceftriaxone $(30 \mu \mathrm{g})$, ceftazidime $(30 \mu \mathrm{g})$, gentamicin $(10 \mu \mathrm{g})$, amikacin $(30 \mu \mathrm{g})$, ciprofloxacin $(5 \mu \mathrm{g})$, nitrofurantoin $(100 \mu \mathrm{g})$, tigecycline $(15 \mu \mathrm{g})$, cotrimoxazole $(25 \mu \mathrm{g})$, piperacillin-tazobactam $(100 / 10 \mu \mathrm{g})$, meropenem $(10 \mu \mathrm{g})$, and imipenem $(10 \mu \mathrm{g})$. The isolates resistant to at least one antibiotic in three or more antimicrobial classes were categorized as MDR, and if they were resistant to at least one agent in all but equal or less than two classes, they were identified as XDR. ${ }^{23}$ Clinical isolates susceptible to first-line antibiotics were excluded for further study while XDR Gram-negative clinical isolates from indoor patients of the hospital were subjected for synergy testing. Ethical approval was obtained from Nepal Health Research Council (Ref No. 270, August 2018) to conduct this study. Organ transplantation at the center is 
done in accordance with the Organ Transplant Act 2055, Transplant legislation 2073 of Nepal. The clinical specimens processed in this study were as part of the routine hospital laboratory procedures.

\section{Synergy Test}

\section{Disk Synergy Method}

XDR clinical isolates of E. coli, Klebsiella spp., Acinetobacter calcoaceticus baumannii complex and Pseudomonas aeruginosa were subjected for disk synergy test by disk diffusion method on MHA using antibiotic disks of amikacin $(30 \mu \mathrm{g})$, ciprofloxacin $(5 \mu \mathrm{g})$, meropenem $(10 \mu \mathrm{g})$ and imipenem $(10 \mu \mathrm{g})$. Combinations of all possible pairs (to which the microorganism was resistant) were tested by placing antibiotic disks at a distance of $20 \mathrm{~mm}$ from each other (center to center). After 16-20 hours of incubation at $35^{\circ} \mathrm{C}$ to $37^{\circ} \mathrm{C}$, if synergy was present among two antibiotics, an inhibition zone was formed between the disks. The criteria for the different results of the combinations were as follows:

Indifferent combinations: Two independent circles around the disks tested within $20 \mathrm{~mm}$ from each other.

Synergy: Enhancement or bridging at or near the junction of the two zones of inhibition.

Antagonism: Truncation near the junction of the two zones of inhibition.

Antibiotic discs of amikacin, ciprofloxacin, colistin were purchased from HiMedia, India, and Meropenem and Imipenem discs from BD, USA.

\section{E-Test MIC:MIC Ratio Method}

The concentration range for ciprofloxacin and meropenem was 0.002 to $32 \mu \mathrm{g} / \mathrm{mL}$, and for colistin sulphate and amikacin, 0.016-256 $\mu \mathrm{g} / \mathrm{mL}$. First of all, MICs were determined for meropenem, amikacin, colistin sulphate and ciprofloxacin against the XDR bacteria by E-test according to the manufacturer's recommendations.

For synergy testing, the bacterial suspension was matched to $0.5 \mathrm{McF}$ arland standard, and with a cotton swab, a lawn culture of bacteria was done on MHA. Meropenem and amikacin, meropenem and ciprofloxacin, amikacin and colistin, amikacin and ciprofloxacin, and meropenem and colistin combinations were used for further studies. E-test strips were placed on different sections of the different MHA plates. The agar was marked with an inoculating loop adjacent to the previously determined MIC value on each strip. For isolates where the MIC exceeded the concentration on the E-test strip, the highest concentration was marked on the agar. The strips were removed and discarded after $1 \mathrm{~h}$ incubation at room temperature. A new antibiotic "B" strip was placed on the area of the previously removed antibiotic " $\mathrm{A}$ " strip so that the antibiotic " $B$ " MIC corresponded with the mark of the antibiotic "A" MIC. The antibiotic " $A$ " E-test strip was applied in a reciprocating fashion to the area of the previous antibiotic " $B$ " strip so that the respective MIC values were aligned. Results for both antimicrobials were read after $16-20 \mathrm{~h}$ incubation in ambient air at $35^{\circ} \mathrm{C}$. The combination MIC was interpreted as the value at which the inhibition zone intersected the scale on each respective E-test strip. E strip results were rounded up to the nearest twofold dilution values for purposes of comparison with broth microdilution MIC results. E-strips of ciprofloxacin, colistin, meropenem were of HiMedia, India, and E-strip of amikacin was from BD, USA.

Finally, synergy, antagonism, indifference, and additivity were determined for each antibiotic in each combination by calculating Fractional Inhibitory Concentration (FIC) as follows:

FIC of antimicrobial " $A$ "= MIC of agent " $A$ " in combination/MIC of agent " $\mathrm{A}$ " alone

FIC of antimicrobial " $\mathrm{B}$ " = MIC of agent " $\mathrm{B}$ " in combination/MIC of agent "B" alone

The total (summation) fractional inhibitory concentration ( $\Sigma$ FIC) or FIC Index for each isolate was calculated according to the following formula:

$\Sigma F I C=$ FIC of antimicrobial 'A' + FIC of antimicrobial 'B'

The results were interpreted as follows: Synergy, if $\Sigma \mathrm{FIC} \leq 0.5$; Indifference, if $1<\Sigma \mathrm{FIC} \leq 4$; Antagonism, if $\Sigma$ FIC $>4$; and Additive, if $0.5<\Sigma$ FIC $\leq 1$.

Phenotypic detection of ESBL and MBL in Gramnegative clinical isolates was also done by double disk method and E-strip method respectively. MIC and FIC were then determined by E-strip MIC:MIC method. Escherichia coli ATCC 25922 and Pseudomonas aeruginosa ATCC 27853 were used as quality control strains for antibiotic sensitivity testing.

One XDR isolate of $P$. aeruginosa was not subjected for synergy testing.

\section{Test for ESBL}

All the strains which showed a diameter of less than $27 \mathrm{~mm}$ for cefotaxime and less than $25 \mathrm{~mm}$ for ceftriaxone, were selected for checking the ESBL production. The ESBL production was tested by the combination disk method by 
using ceftazidime $(30 \mu \mathrm{g})$ and ceftazidime-clavulanate (20/ $10 \mu \mathrm{g}$ ) discs. ${ }^{9}$ The organisms positive for ESBL showed an increase of $\geq 5 \mathrm{~mm}$ zone of inhibition with clavulanic acid as compared to the zone size for ceftazidime alone. ${ }^{22}$

\section{Test for MBL}

To screen for the presence of MBLs, E-test strips (AB Biodisk, Sweden) were used according to the manufacturer's instructions. E-test MBL strips contain increasing concentrations of imipenem (IP) on one end and imipenem overlaid with EDTA (IPI) on the other. A reduction in the imipenem MIC in the presence of EDTA of greater than or equal to eight-fold (IP/IPI $\geq 8$ ) was interpreted as MBLpositive. $^{24}$

Data were recorded in the proforma sheet (all sample isolates were encoded) and statistical analyses were done by using MS Excel.

\section{Results}

Out of total of 1155 clinical samples processed for culture, 308 samples yielded microbial growth. Amongst those 308 culture-positive samples, $142(46.10 \%)$ were E. coli which was the commonest bacteria, 76 (24.68\%) were K. pneumoniae, 27 (8.77\%) were Acinetobacter, 24 $(7.79 \%)$ were $P$. aeruginosa and the remaining 39 $(12.66 \%)$ were other miscellaneous bacteria. Multidrugresistance was observed in $21.43 \%(n=66)$ of the total isolates. Similarly, 31 isolates were XDR which accounted for $10.06 \%(31 / 308)$ of the total isolates, and 46.97\% (31/ 66) of the MDR isolates. No pandrug-resistant (PDR) isolate was detected. The number and percentage of "MDR", "XDR only", and "non-MDR" isolates among various organisms are shown in the table (Table 1).

Amongst the 66 MDR clinical isolates of various bacteria, E. coli accounted for the largest fraction which was $51.52 \%$ (34 out of 66 ), followed by $K$. pneumoniae which was $36.36 \%$
(24 out of 66), Acinetobacter 9.09\% (6 out of 66) and Pseudomonas 3.03\% (2 out of 66). Similarly, out of 31 XDR isolates, $45.16 \%$ were detected each from $E$. coli and $K$. pneumoniae (14 each out of 31 ), followed by Acinetobacter which comprised $6.45 \%$ (2 out of 31 ) and $P$. aeruginosa which comprised $3.22 \%$ (1 out of 31 ) of the XDR isolates.

The number and percentage of ESBL and MBL among all culture-positive isolates are also shown in the table (Table 1).

Antibiotic resistance pattern of MDR clinical isolates of E. coli, Klebsiella, Pseudomonas, and Acinetobacter is presented in the table (Table 2).

Further, antibiotic synergy was tested in 30 XDR clinical isolates using various antibiotic combinations among which $14 \mathrm{XDR}$ isolates each of $E$. coli and $K$. pneumoniae and 2 isolates of $A c b$ complex were used. The result of antibiotic synergy testing using various antibiotic combinations in these XDR isolates is shown in Table 3, and that in individual XDR isolates are shown in the subsequent tables (Tables 4, Table 5, Table 6).

\section{Discussion}

The global rise in antimicrobial resistance has triggered the adoption of different strategies to combat such situation, one of them being the use of combination therapy wherein one antimicrobial can synergize the efficacy of the other. To the best of our knowledge, this is the first study on antimicrobial synergy testing from Nepal.

Among all positive growths in culture, the most common organism was $E$. coli followed by $K$. pneumoniae, $A c b$ complex, P. aeruginosa and miscellaneous bacteria. The fact that E. coli, Klebsiella are among the most common organisms isolated in clinical specimens is wellknown and has been shown by several studies done in the past, ${ }^{25-27}$ and the importance of these organisms as

Table I Number and Percentage of MDR, XDR Only, Non-MDR, ESBL and MBL Positive Isolates Among All Culture Positive Isolates

\begin{tabular}{|l|c|c|c|c|c|c|}
\hline \multirow{2}{*}{ Total Isolates } & MDR & XDR Only & Non-MDR & ESBL & MBL \\
\cline { 3 - 7 } & & Number (\%) & Number (\%) & Number (\%) & Number (\%) & Number (\%) \\
\hline E. coli & 142 & $34(23.94)$ & $14(9.86)$ & $108(76.06)$ & $12(8.45)$ & $10(7.04)$ \\
Klebsiella & 76 & $24(31.58)$ & $14(18.42)$ & $52(68.42)$ & $2(2.63)$ & $7(9.21)$ \\
Pseudomonas & 24 & $2(8.33)$ & $1(4.17)$ & $22(91.67)$ & $0(0.00)$ & $0(0.00)$ \\
Acinetobacter & 27 & $6(22.22)$ & $2(7.41)$ & $21(77.78)$ & $0(0.00)$ & $1(3.70)$ \\
Others & 39 & $0(0.00)$ & $0(0.00)$ & $39(100)$ & $0(0.00)$ & $0(0.00)$ \\
Total & 308 & $66(21.43)$ & $31(10.06)$ & $242(78.57)$ & $14(4.54)$ & $18(5.84)$ \\
\hline
\end{tabular}


Table 2 Resistance Pattern of MDR E. coli, K. pneumoniae, Acinetobacter spp. and P. aeruginosa

\begin{tabular}{|c|c|c|c|c|}
\hline \multirow[t]{2}{*}{ Antimicrobials } & \multicolumn{4}{|c|}{ Resistance Pattern of Various Organisms } \\
\hline & $\begin{array}{l}\text { E. coli }(n=34) \\
\text { Number }(\%)\end{array}$ & $\begin{array}{c}\text { K. pneumoniae }(n=24) \\
\text { Number }(\%)\end{array}$ & $\begin{array}{c}\text { Acinetobacter spp. }(\mathrm{n}=6) \\
\text { Number }(\%)\end{array}$ & $\begin{array}{c}\text { P. aeruginosa }(\mathrm{n}=2) \\
\text { Number }(\%)\end{array}$ \\
\hline Gentamicin & $21(61.76)$ & $16(66.67)$ & $6(100)$ & $0(0.00)$ \\
\hline Amikacin & $16(47.06)$ & $14(58.33)$ & $4(66.67)$ & I (50.00) \\
\hline Meropenem & $21(61.76)$ & $18(75.00)$ & $6(100)$ & I $(50.00)$ \\
\hline Imipenem & $21(61.76)$ & $15(62.50)$ & $4(66.67)$ & I (50.00) \\
\hline Ceftazidime & $27(79.4 I)$ & $23(95.83)$ & $6(100)$ & $2(100)$ \\
\hline Ceftriaxone & $32(94.12)$ & $24(100)$ & $6(100)$ & - \\
\hline Ciprofloxacin & $33(97.06)$ & $24(100)$ & $6(100)$ & $2(100)$ \\
\hline Cotrimoxazole & $33(97.06)$ & $23(95.83)$ & $6(100)$ & - \\
\hline Tigecycline & $5(\mid 4.7 I)$ & $5(20.83)$ & $6(100)$ & - \\
\hline Amoxycillin & $34(100)$ & - & - & - \\
\hline Piperacillin-tazobactam & I8 (52.94) & $18(75.00)$ & $5(83.33)$ & I (50.00) \\
\hline Chloramphenicol & $27(79.4 I)$ & $24(100)$ & - & - \\
\hline Polymyxin B & $0(0.00)$ & I (4.17) & $0(0.00)$ & $0(0.00)$ \\
\hline Colistin sulphate & $0(0.00)$ & I (4.17) & $0(0.00)$ & $0(0.00)$ \\
\hline Nitrofurantoin & $6(22.22)$ & I8 (85.7I) & - & - \\
\hline
\end{tabular}

Table 3 Antibiotic Synergy Testing Using Various Antibiotic Combinations in Clinical Isolates $(n=30)$

\begin{tabular}{|l|c|c|c|c|}
\hline Antibiotic Combination & $\begin{array}{c}\text { Synergy } \\
\text { Number (\%) }\end{array}$ & $\begin{array}{c}\text { Additive } \\
\text { Number (\%) }\end{array}$ & $\begin{array}{c}\text { Indifference } \\
\text { Number (\%) }\end{array}$ & $\begin{array}{c}\text { Antagonism } \\
\text { Number (\%) }\end{array}$ \\
\hline Meropenem + Amikacin & $2(6.66 \%)$ & $11(36.67 \%)$ & $17(56.67 \%)$ & $0(0.00 \%)$ \\
Meropenem + Ciprofloxacin & $0(0.00 \%)$ & $0(0.00 \%)$ & $30(100 \%)$ & $0(0.00 \%)$ \\
Amikacin + Colistin & $5(16.67 \%)$ & $8(26.67 \%)$ & $15(50.00 \%)$ & $2(6.66 \%)$ \\
Amikacin + Ciprofloxacin & $2(6.67 \%)$ & $2(6.67 \%)$ & $24(80.00 \%)$ & $2(6.66 \%)$ \\
Meropenem + Colistin & $7(23.33 \%)$ & $6(20.00 \%)$ & $13(43.34 \%)$ & $4(13.33 \%)$ \\
\hline
\end{tabular}

Table 4 Antibiotic Synergy Testing in XDR E. coli $(n=14)$

\begin{tabular}{|l|c|c|c|c|}
\hline Antibiotic Combination & $\begin{array}{c}\text { Synergy } \\
\text { Number (\%) }\end{array}$ & $\begin{array}{c}\text { Additive } \\
\text { Number (\%) }\end{array}$ & $\begin{array}{c}\text { Indifference } \\
\text { Number (\%) }\end{array}$ & $\begin{array}{c}\text { Antagonism } \\
\text { Number (\%) }\end{array}$ \\
\hline Meropenem + Amikacin & $0(0.00 \%)$ & $4(28.57 \%)$ & $10(71.43 \%)$ & $0(0.00 \%)$ \\
Meropenem + Ciprofloxacin & $0(0.00 \%)$ & $0(0.00 \%)$ & $14(100 \%)$ & $0(0.00 \%)$ \\
Amikacin + Colistin & $0(0.00 \%)$ & $2(14.29 \%)$ & $10(71.42 \%)$ & $2(14.29 \%)$ \\
Amikacin + Ciprofloxacin & $2(14.29 \%)$ & $0(0.00 \%)$ & $10(71.42 \%)$ & $2(14.29 \%)$ \\
Meropenem + Colistin & $4(28.57 \%)$ & $2(14.29 \%)$ & $4(28.57 \%)$ & $4(28.57 \%)$ \\
\hline
\end{tabular}

Table 5 Antibiotic Synergy Testing in XDR K. pneumoniae $(n=14)$

\begin{tabular}{|l|c|c|c|c|}
\hline Antibiotic Combination & $\begin{array}{c}\text { Synergy } \\
\text { Number (\%) }\end{array}$ & $\begin{array}{c}\text { Additive } \\
\text { Number (\%) }\end{array}$ & $\begin{array}{c}\text { Indifference } \\
\text { Number (\%) }\end{array}$ & $\begin{array}{c}\text { Antagonism } \\
\text { Number (\%) }\end{array}$ \\
\hline Meropenem + Amikacin & $2(14.28 \%)$ & $6(42.86 \%)$ & $6(42.86 \%)$ & $0(0.00 \%)$ \\
Meropenem + Ciprofloxacin & $0(0.00 \%)$ & $0(0.00 \%)$ & $14(100 \%)$ & $0(0.00 \%)$ \\
Amikacin + Colistin & $4(28.57 \%)$ & $6(42.86 \%)$ & $4(28.57 \%)$ & $0(0.00 \%)$ \\
Amikacin + Ciprofloxacin & $0(0.00 \%)$ & $2(14.29 \%)$ & $12(85.71 \%)$ & $0(0.00 \%)$ \\
Meropenem + Colistin & $2(14.29 \%)$ & $4(28.57 \%)$ & $8(57.14 \%)$ & $0(0.00 \%)$ \\
\hline
\end{tabular}


Table 6 Antibiotic Synergy Testing in XDR Acb Complex $(n=2)$

\begin{tabular}{|l|c|c|c|c|}
\hline Antibiotic Combination & $\begin{array}{c}\text { Synergy } \\
\text { Number (\%) }\end{array}$ & $\begin{array}{c}\text { Additive } \\
\text { Number (\%) }\end{array}$ & $\begin{array}{c}\text { Indifference } \\
\text { Number (\%) }\end{array}$ & $\begin{array}{c}\text { Antagonism } \\
\text { Number (\%) }\end{array}$ \\
\hline Meropenem + Amikacin & $0(0.00 \%)$ & $1(50.00 \%)$ & $I(50.00 \%)$ & $0(0.00 \%)$ \\
Meropenem + Ciprofloxacin & $0(0.00 \%)$ & $0(0.00 \%)$ & $2(100 \%)$ & $0(0.00 \%)$ \\
Amikacin + Colistin & $I(50.00 \%)$ & $0(0.00 \%)$ & $1(50.00 \%)$ & $0(0.00 \%)$ \\
Amikacin + Ciprofloxacin & $0(0.00 \%)$ & $0(0.00 \%)$ & $2(100 \%)$ & $0(0.00 \%)$ \\
Meropenem + Colistin & $I(50.00 \%)$ & $0(0.00 \%)$ & $1(50.00 \%)$ & $0(0.00 \%)$ \\
\hline
\end{tabular}

emerging global threat has been presented in several studies done now and then. ${ }^{26,28-30}$

In this study, a significant proportion of the isolates were MDR and among those too, a large fraction were XDR isolates; however, no PDR isolates were found. The members of Enterobacteriaceae (K. pneumoniae and E. coli), were found to be more MDR and XDR in frequency as compared to Acinetobacter, and Pseudomonas isolates. Similar findings were seen in some other studies as well. ${ }^{26,27,31}$

ESBL- and MBL-producing bacteria, particularly $K$. pneumoniae and E. coli, are a matter of concern worldwide these days. In our study, among the total culturepositive isolates, $4.5 \%$ were ESBL-producers and 5.8\% were MBL-producers. Among the various bacteria, the highest proportion of ESBL-producers was seen in $E$. coli whereas a similar proportion of MBL-producers was seen in K. pneumoniae. Regarding Acinetobacter spp., MBL-production was seen in few isolates, but none were producing ESBL. Several reports from different parts of the world in the recent past have also shown a significant proportion of clinical isolates producing ESBL and MBL. ${ }^{27,31-33}$

Various antibiotics were used as the first-line and the second-line agents for antibiotic susceptibility testing for all culture-positive Gram-negative isolates. The sensitivity pattern of these bacterial isolates to various antibiotics has shown a wide range of results from $100 \%$ resistance to some of the antibiotics to $100 \%$ susceptibility to some others. A large proportion of MDR isolates were found to be sensitive to few antibiotics including, colistin, polymyxin B, and tigecycline. All of the MDR E. coli isolates were sensitive to polymyxin B and colistin, and a high number of them were sensitive to tigecycline. A significant proportion of MDR E. coli were also susceptible to nitrofurantoin followed by amikacin and piperacillin-tazobactam. All of the MDR E. coli isolates were resistant to amoxicillin, a large proportion resistant to cotrimoxazole, ciprofloxacin, and ceftriaxone, and a huge proportion were found to be resistant to both carbapenems (meropenem and imipenem) and gentamicin. This result has supported the trend of emerging antimicrobial resistance among $E$. coli to various antibiotics as has also been shown by various other studies. ${ }^{34}$ A study done in northeast Ethiopia has also shown high rates of antimicrobial resistance in $E$. coli to amoxicillin and tetracycline whereas a significantly high degree of sensitivity rates to nitrofurantoin (96.4\%), gentamicin (79.6\%), and ciprofloxacin. ${ }^{35}$ In our study, a high degree of sensitivity was seen with nitrofurantoin but not with gentamicin and ciprofloxacin, suggesting an increasing trend of resistance to gentamicin and ciprofloxacin too in our context.

Similarly, high sensitivity to polymyxin B, colistin, and tigecycline was also seen with MDR $K$. pneumoniae isolates in this study, whereas as high as $100 \%$ of isolates were found to be resistant to ceftriaxone, ciprofloxacin, and chloramphenicol, with a large proportion being resistant to several other antibiotics like ceftazidime, cotrimoxazole, nitrofurantoin, meropenem, imipenem, piperacillin-tazobactam, gentamicin, and amikacin. Likewise, high degree resistance to antibiotics like ciprofloxacin and third-generation cephalosporins was seen in a study from Taiwan. ${ }^{36}$ However, another study done over a period of twenty-one years and published recently has shown that more than $50 \%$ of Klebsiella isolates to be susceptible to several antibiotics like levofloxacin, piperacillin-tazobactam, meropenem and imipenem, unlike our findings. ${ }^{37}$

In our study, $100 \%$ of MDR $P$. aeruginosa isolates have shown susceptibility to gentamicin, polymyxin B, and colistin whereas they were found to be resistant to ceftazidime and ciprofloxacin suggesting the increasing trend of antimicrobial resistance to some antimicrobials that were once effective against Pseudomonas. Fifty percent of MDR Pseudomonas isolates were sensitive to amikacin, carbapenems, and antipseudomonal penicillin (piperacillin-tazobactam). This has supported the 
fact that polymyxins are still among the few of the lastresort drugs to combat MDR Pseudomonas spp. The finding also suggests that aminoglycoside(s) can be one of the agents of choice in the empirical management of cases of suspected pseudomonal infection. Some other studies have also demonstrated a very good sensitivity of Pseudomonas to carbapenems and some of the aminoglycosides including amikacin. ${ }^{38,39}$

Sensitivity of MDR Acinetobacter spp. was also found to be $100 \%$ with polymyxin B and colistin; however, they were found to be resistant to a majority of antibiotics, viz., gentamicin, meropenem, ceftazidime, ceftriaxone, tigecycline, and cotrimoxazole. A small fraction was however sensitive to amikacin, imipenem, and piperacillintazobactam.

Among the combinations of antibiotics, meropenem +colistin has shown the highest proportion of "synergy" among all XDR isolates followed by amikacin+colistin. The highest degree of "additivity" has been shown by the antibiotic combination of meropenem+amikacin followed by amikacin+colistin and then meropenem+colistin. This result has suggested a possible beneficial action while combining meropenem, amikacin, and colistin with one another. Moreover, multidrug therapy especially colistin combinations have been used increasingly as the lastresort treatment for MDR strains especially in the empiric management of Gram-negative sepsis, and has been proven beneficial by several studies done in the recent past. ${ }^{40-43}$ All of the XDR isolates showed "indifference" to the combination of meropenem+ciprofloxacin whereas few isolates showed "antagonism" when tested with meropenem+colistin, amikacin+colistin, and amikacin+ciprofloxacin. No "antagonism" was seen with meropenem tamikacin.

Among the synergy tests done with XDR E. coli, only meropenem+colistin and amikacin+ciprofloxacin showed "synergy". "Additivity" was seen when two among the three antibiotics, viz., meropenem, colistin, and amikacin were combined. This further strengthened the possibility of beneficial action while combining meropenem, amikacin, and colistin with one another in pair. The majority of the rest showed indifference.

When tested with XDR K. pneumoniae, the same three antibiotics (meropenem, amikacin and colistin), when combined with one another in pair showed some degree of "synergy" and "additivity". A small proportion showed "additivity" with amikacin+ciprofloxacin too. All the rest showed indifference. None of the test results showed "antagonism" in case of $K$. pneumoniae. Half of the tests with XDR $A c b$ complex resulted in "synergy" when colistin was combined with either meropenem or amikacin. Meropenem when combined with amikacin showed an "additive" result in 50\% XDR $A c b$ complex isolates. This also supported the finding that meropenem, amikacin, and colistin when combined with one another might have a beneficial action. None of the tests resulted in "antagonism" with the XDR $A c b$ complex. A recent study has also found colistin+meropenem to have an additive effect against most of the carbapenem-resistant (CR) A. baumannii isolates (83.3\%), and no antagonism for this combination. ${ }^{44}$ Among the CR P. aeruginosa isolates tested, the colistin+meropenem showed synergistic effects against $63.6 \%$ isolates and additive effect in $36.4 \%$ isolates. ${ }^{44}$ Hence the combination of meropenem and colistin can be used as the potential therapy against infection with such XDR pathogens.

\section{Conclusion}

This study has shown a large proportion of clinical isolates to be MDR and XDR among which E. coli and $K$. pneumoniae are the most common ones followed by Acinetobacter and Pseudomonas. This study has suggested a possible beneficial action while combining meropenem, amikacin, and colistin with one another in pair. Further studies with a larger sample size and a variety of synergy testing methods are required to generalise these findings.

\section{Abbreviations}

MDR, multidrug-resistant; XDR, extensively drugresistant; ESBLs, extended-spectrum beta lactamases; MBLs, metallo-beta-lactamases; AMR, antimicrobial resistance; KPC, Klebsiella pneumoniae carbapenemase; WHO, World Health Organisation; e-strip test, epsilometer- strip test; MIC, minimum inhibitory concentration; CL, colistin; CIP, ciprofloxacin; Ak, amikacin; MEM, meropenem; HOTC, Human Organ Transplant Centre; MHA, Mueller-Hinton Agar; CLSI, Clinical and Laboratory Standards Institute; FIC, fractional inhibitory concentration; IP, imipenem; E. coli, Escherichia coli; K. pneumoniae, Klebsiella pneumoniae; P. aeruginosa, Pseudomonas aeruginosa; Acb complex, Acinetobacter calcoaceticus baumannii complex; PDR, pandrugresistant; CR, Carbapenem-resistant.

\section{Acknowledgments}

We would like to acknowledge all the patients who provided their samples for this study, the laboratory and 
clinical staffs of Shahid Dharmabhakta National Transplant Center; and the laboratory science faculties of Janamaitri Foundation Institute of Health Sciences.

\section{Disclosure}

The authors report no conflicts of interest in this work.

\section{References}

1. Aminov RI. A brief history of the antibiotic era: lessons learned and challenges for the future. Front Microbiol. 2010;1:134. doi:10.3389/ fmicb.2010.00134

2. Gottlieb T, Nimmo GR. Antibiotic resistance is an emerging threat to public health: an urgent call to action at the Antimicrobial Resistance Summit 2011. Med J Aust. 2011;194(6):281-283. doi:10.5694/ j.1326-5377.2011.tb02973.x

3. Siegel RE. Emerging gram-negative antibiotic resistance: daunting challenges, declining sensitivities, and dire consequences. Respir Care. 2008;53(4):471-479.

4. Roca I, Akova M, Baquero F, et al. The global threat of antimicrobial resistance: science for intervention. New Microbes New Infect. 2015;6:22-29. doi:10.1016/j.nmni.2015.02.007

5. Rawat D, Extended-spectrum ND. $\beta$-lactamases in gram negative bacteria. J Glob Infect Dis. 2010;2(3):263-274. doi:10.4103/0974777X.68531

6. Palzkill T. Metallo- $\beta$-lactamase structure and function. Ann $N$ Y Acad Sci. 2013;1277:91-104. doi:10.1111/j.1749-6632.2012.06796.x

7. Jacoby GA. AmpC beta-lactamases. Clin Microbiol Rev. 2009;22 (1):161-182. doi:10.1128/CMR.00036-08

8. Queenan AM, Bush K. Carbapenemases: the versatile beta-lactamases. Clin Microbiol Rev. 2007;20(3):440-458. doi:10.1128/CMR.00001-07

9. Cantón R, González-Alba JM, Galán JC. CTX-M Enzymes:Origin and Diffusion. Front Microbiol. 2012;3:110. doi:10.3389/fmicb. 2012.00110

10. Rice LB. The clinical consequences of antimicrobial resistance. Curr Opin Microbiol. 2009;12(5):476-481. doi:10.1016/j.mib.2009.08.001

11. Chi H, Holo H. Synergistic antimicrobial activity between the broad spectrum bacteriocin garvicin KS and Nisin, Farnesol and Polymyxin B against gram-positive and gram-negative bacteria. Curr Microbiol. 2018;75(3):272-277. doi:10.1007/s00284-017-1375-y

12. Tallarida RJ. Quantitative methods for assessing drug synergism. Genes Cancer. 2011;2(11):1003-1008. doi:10.1177/194760191244 0575

13. Gunnison JB, Shevky MC, Bruff JA, Coleman VR, Jawetz E. Studies on antibiotic synergism and antagonism: the effect in vitro of combinations of antibiotics on bacteria of varying resistance to single antibiotics. J Bacteriol. 1953;66(2):150-158. doi:10.1128/JB.66.2. 150-158.1953

14. Doern CD. When does 2 plus 2 equal 5? A review of antimicrobial synergy testing. $J$ Clin Microbiol. 2014;52(12):4124-4128. doi:10. 1128/JCM.01121-14

15. Eliopoulos GM, Moellering JRC. Antibiotic synergism and antimicrobial combinations in clinical infections. Rev Infect Dis. 1982;4 (2):282-293. doi:10.1093/clinids/4.2.282

16. Gales AC, Jones RN, Sader HS. Global assessment of the antimicrobial activity of polymyxin B against 54731 clinical isolates of Gram-negative bacilli: report from the SENTRY antimicrobial surveillance programme (2001-2004). Clin Microbiol Infect. 2006;12 (4):315-321. doi:10.1111/j.1469-0691.2005.01351.x

17. Percin D, Akyol S, Kalin G. In vitro synergism of combinations of colistin with selected antibiotics against colistin-resistant Acinetobacter baumannii. GMS Hyg Infect Control. 2014;9(2): Doc14. doi:10.3205/dgkh000234
18. Fantin B, Carbon C. In vivo antibiotic synergism: contribution of animal models. Antimicrob Agents Chemother. 1992;36(5):907-912. doi:10.1128/AAC.36.5.907

19. WHO Publishes List of Bacteria for Which New Antibiotics are Urgently Needed. WHO News Release; Geneva. 2017.

20. Kasper D, Fauci A, Hauser S, Longo D, Jameson J, Loscalzo J, Eds. Harrison's Principles of Internal Medicine. Mcgraw-hill; 2015:19e.

21. Isenberg H, ed. Clinical Microbiology Procedures Handbook. Washington, DC: American Society for Microbiology (ASM); 2007.

22. Patel JB, Cockerill FR, Eliopoulos GM, et al. CLSI. Performance Standards for Antimicrobial Susceptibility Testing, Supplement M100S, $26^{\text {th }}$ ed. Wayne PA; 2016,

23. Magiorakos AP, Srinivasan A, Carey RB. Multidrug-resistant, extensively drug-resistant and pandrug-resistant bacteria: an international expert proposal for interim standard definitions for acquired resistance. Clin Microbiol Infect. 2012;18(3):268-281. doi:10.1111/ j.1469-0691.2011.03570.x

24. Segal H, Elisha BG. Use of Etest MBL strips for the detection of carbapenemases in Acinetobacter baumannii. $J$ Antimicrob Chemother. 2005;56(3):598. doi:10.1093/jac/dki265

25. Daoud Z, Afif C. Escherichia coli isolated from urinary tract infections of lebanese patients between 2000 and 2009: epidemiology and profiles of resistance. Chemother Res Pract. 2011;2011:218431. doi:10.1155/2011/218431

26. Basak S, Singh P, Rajurkar M. Multidrug resistant and extensively drug resistant bacteria: a study. J Pathog. 2016;2016:4065603. doi: $10.1155 / 2016 / 4065603$

27. Nepal K, Pant ND, Neupane B, et al. Extended spectrum beta-lactamase and metallo beta-lactamase production among Escherichia coli and Klebsiella pneumoniae isolated from different clinical samples in a tertiary care hospital in Kathmandu, Nepal. Ann Clin Microbiol Antimicrob. 2017;16(1):62. doi:10.1186/s12941-0170236-7

28. Gandra S, Tseng KK, Arora A, et al. The mortality burden of multidrug-resistant pathogens in India: a retrospective, observational study. Clin Infect Dis. 2019;69(4):563-570. doi:10.1093/cid/ciy955

29. Eichenberger EM, Thaden JT. Epidemiology and mechanisms of resistance of extensively drug resistant gram-negative bacteria. Antibiotics (Basel). 2019;8(2):37. doi:10.3390/antibiotics8020037

30. Souli M, Galani I, Giamarellou H. Emergence of extensively drug-resistant and pandrug-resistant Gram-negative bacilli in Europe. Euro Surveill. 2008;13(47):19045. doi:10.2807/ese.13.47.19045-en

31. Ghadri H, Vaez H, Razavi-Azarkhiavi K, et al. Prevalence and antibiotic susceptibility patterns of extended-spectrum Blactamase and metallo- $\beta$-lactamase-producing uropathogenic Escherichia coli isolates. Lab Med. 2014;45(4):291-296. doi:10.1309/LMHEP4VQHEY2POOK

32. Ugwu MC, Igbokwe JO, Okezie U, et al. Prevalence of ESBLs and MBLs among Escherichia coli and Klebsiella pneumoniae isolates from a Nigerian Abattoir. J Trop Dis. 2018;6:2. doi:10.4172/2329891X.1000261

33. Mishra SK, Shrestha R, Rijal B, et al. The bad, the ugly and the demon: a tale of extensively drug-resistant, extended-spectrum-betalactamase- and metallo-beta-lactamase-producing superbugs associated with nosocomial pneumonia. Asian Pac J Trop Dis. 2015;5 (1):71-76. doi:10.1016/S2222-1808(14)60630-7

34. Kulkarni SR, Peerapur BV, Sailesh KS. Isolation and antibiotic susceptibility pattern of Escherichia coli from urinary tract infections in a Tertiary Care Hospital of North Eastern Karnataka. J Nat Sci Biol Med. 2017;8(2):176-180. doi:10.4103/0976-9668.210012

35. Kibret M, Abera B. Antimicrobial susceptibility patterns of E. coli from clinical sources in northeast Ethiopia. Afr Health Sci. 2011;11 (Suppl 1):S40-5. doi:10.4314/ahs.v11i3.70069

36. Lin WP, Wang JT, Chang SC, et al. The antimicrobial susceptibility of Klebsiella pneumoniae from Community Settings in Taiwan, a Trend Analysis. Sci Rep. 2016;6:36280. doi:10.1038/srep36280 
37. Srinivasan S, Saldanha J, Abhyankar S, et al. Antibiotic sensitivity pattern of Klebsiella species in burn wounds at Bai Jerbai Wadia hospital for children, Mumbai, India-a 21 year study. Int $J$ Burns Trauma. 2017;7(5):64-71.

38. Javiya VA, Ghatak SB, Patel KR, et al. Antibiotic susceptibility patterns of Pseudomonas aeruginosa at a tertiary care hospital in Gujarat, India. Indian $J$ Pharmacol. 2008;40(5):230-234. doi: $10.4103 / 0253-7613.44156$

39. Ahmed OB. Incidence and antibiotic susceptibility pattern of pseudomonas aeruginosa isolated from inpatients in two Tertiary Hospitals. Clin Microbiol. 2016;5:2. doi:10.4172/2327-5073.1000235

40. Safdar N, Handelsman J, Maki DG. Does combination antimicrobial therapy reduce mortality in Gram-negative bacteraemia? A meta-analysis. Lancet Infect Dis. 2004;4(8):519-527. doi:10.1016/ S1473-3099(04)01108-9

41. Kumar A, Safdar N, Kethireddy S, et al. A survival benefit of combination antibiotic therapy for serious infections associated with sepsis and septic shock is contingent only on the risk of death: a meta-analytic/meta-regression study. Crit Care Med. 2010;38 (8):1651-1664. doi:10.1097/CCM.0b013e3181e96b91
42. Tamma PD, Cosgrove SE, Maragakis LL. Combination therapy for treatment of infections with gram-negative bacteria. Clin Microbiol Rev. 2012;25(3):450-470. doi:10.1128/CMR.05041-11

43. Traugott KA, Echevarria K, Maxwell P, et al. Monotherapy or combination therapy? The Pseudomonas aeruginosa conundrum. Pharmacotherapy. 2011;31(6):598-608. doi:10.1592/phco.31.6.598

44. Ramadan RA, Gebriel MG, Kadry HM, et al. Carbapenem-resistant Acinetobacter baumannii and Pseudomonas aeruginosa: characterization of carbapenemase genes and E-test evaluation of colistin-based combinations. Infect Drug Resist. 2018;11:1261-1269. doi:10.2147/ IDR.S170233
Infection and Drug Resistance

\section{Publish your work in this journal}

Infection and Drug Resistance is an international, peer-reviewed openaccess journal that focuses on the optimal treatment of infection (bacterial, fungal and viral) and the development and institution of preventive strategies to minimize the development and spread of resistance. The journal is specifically concerned with the epidemiology of
Dovepress

antibiotic resistance and the mechanisms of resistance development and diffusion in both hospitals and the community. The manuscript management system is completely online and includes a very quick and fair peerreview system, which is all easy to use. Visit http://www.dovepress.com/ testimonials.php to read real quotes from published authors. 